\title{
Bioluminescence: Chemical Study on Visible Light Emission from Fungal Mycelium and Fruiting Body
}

\author{
Katsunori Teranishi* \\ Graduate School of Bioresources, Mie University, Japan
}

Submission: March 19, 2018; Published: March 28, 2018

*Corresponding author: Katsunori Teranishi, Graduate School of Bioresources, Mie University, 1577 Kurimamachiya, Tsu, Mie, Japan, Tel: +81592319615; Email: teranisi@bio.mie-u.ac.jp

\begin{abstract}
Although the visible light emission by living organisms is generally thought to be rare, in fact the bioluminescent phenomenon can be widely observed in nature, for examples insects, fishes, bacteria, and fungi. The phenomenon has intrigued scientific researchers over the years. The chemical mechanisms underlying bioluminescence differ between species. Some mechanisms, such as bioluminescence of firefly, jellyfish, bacteria, and dinoflagellate, have been elucidated and further more their principles have allowed the development of many novel technologies in agriculture, biology, ecology, and medicine. However, many other bioluminescence mechanisms yet remain to be understood. Further research of bioluminescence will lead discoveries of biological significance and novel possibility in science. Here I present studies on chemical mechanism underlying fungal bioluminescence, which are actively in progress.
\end{abstract}

Keywords: Bioluminescence; Chemiluminescence; Fungus; Luciferin; Luciferase; Mechanism

\section{Mini Review}

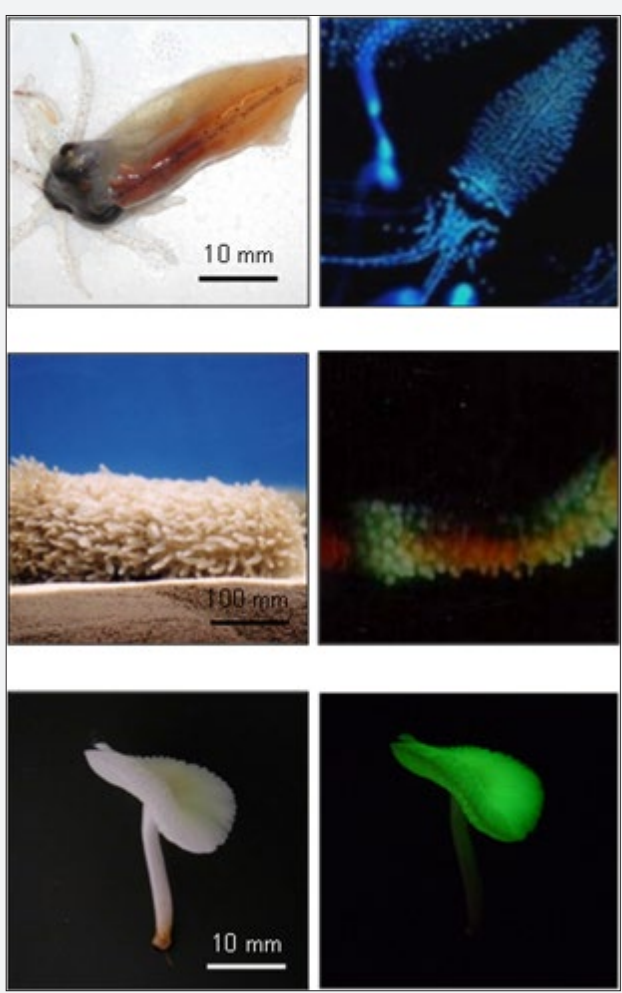

Figure 1: Bioluminescent firefly squid (Watasenia scintillans) (top), sea pen (Cavernularia obesa) (center), and fungus (Mycena chlorophos) (bottom). Left photos are under the white light, and right photos are in the dark.
Bioluminescence is the emission of visible light by living organisms, such as bacteria, fireflies, fishes, ostracods of the genus Cypridina, and jellyfish of the genus Aequorea [1]. The bioluminescence phenomenon has been arousing fascination in human and curiosity in scientists from a long time ago (Figure 1). Detailed chemical principles in bioluminescence have been disclosed by biological, organic, and physical chemists since 1950 's, and then it has been understood that the bioluminescence phenomenon is caused by energy conversion of chemical energy to light energy via chemical reactions in bioluminescence systems. Moreover, the bioluminescence principles have been applied in many scientific fields such as agriculture, biology, ecology, and medicine [2]. Since instrumentation for measuring light is very sensitive and low background, chemiluminescence methods based on the bioluminescence principles has come into widespread use for quantitative determination of specific substances in many scientific fields. Bioluminescent organisms use their light, which possesses various colors, periodic patterns, and intensities, for self-defense against predators, camouflage, intra specific communication, or attracting mates or prey [3].

Fungal bioluminescence is also widely observed on decaying wood or leaves in night. Thus, the phenomenon is called "foxfire" or "shining wood". The total number of documented species of luminous fungi by 2016 is more than 80 [4]. Fungal bioluminescence, which varies among species, occurs in the basidiocarp, mycelium, spores, or in 
their combinations [5-7]. Although fungal bioluminescence generally shows lower intensities than other bioluminescent organisms such as fireflies and ostracods, the light emission from fungi is continuously generated for day and night. Thus, total light emission from certain bioluminescent fungi may be comparable with that of firefly. The color of fungal bioluminescence is commonly green. With regard to functions of fungal bioluminescence, Olivera et al. reported that fruiting bodies of bioluminescent fungus Neonothopanus gardneri can attract potential spore dispersing insects by glowing [8]. On the other hand, Weinstein et al. reported that Australian fruiting bodies of bioluminescent fungus Omphalotus nidiformis did not attract the insects [9]. The mycelium is underground or inside a decaying wood. It is possible that fungal bioluminescence is a by-product of metabolism, and possesses no function. Functions of fungal bioluminescence yet remain to be understood.

In spite of the wide popularity of fungal bioluminescence, chemical mechanism underlying fungal bioluminescence has been subjected to far less scientific investigation than the bioluminescence from other sources. In 1668, Boyle reported that air was needed for the light emission of shining wood [10]. Although many researchers studied on chemical mechanism underlying fungal bioluminescence, their efforts were unsuccessful until Airth's group showed positive results using bioluminescent fungi Collybia velutipes and Armillariamelleain 1959 [11]. Airth et al. reported that the bioluminescence was $\mathrm{NAD}(\mathrm{P}) \mathrm{H}$-dependent luminescence reaction, in which unknown substrate (luciferin), molecular oxygen, water-soluble enzyme, and water-insoluble enzyme (luciferase) were involved [1113]. However, after that, the luciferase and luciferin were not isolated and their structures and luminescent properties were not determined. Kuwabara and Wassink isolated a luciferin, which displayed chemiluminescence in the presence of $\mathrm{H}_{2} \mathrm{O}_{2}$, in a crystal form from the mycelia of Omphalia flavida [14]; however, its chemical structure was not reported.

Shimomura demonstrated a negative result with regard to the Airth's luciferin-luciferase reaction to the fruiting body bioluminescence of the luminous fungus Panellus stipticus; the precursors, panal, PS-A, and PS-B (Figure 2), of light-emitting compounds were isolated from the luminous fruiting bodies that upon activation with ammonium or primary amines, emitted light in the presence of $\mathrm{H}_{2} \mathrm{O}_{2}$ and $\mathrm{Fe}^{2+}$ or in the presence of superoxide anion and molecular oxygen $[15,16]$. Moreover, Shimomura purified the chemiluminescent activation products that were produced by reaction of the precursors with methylamine, and inferred the possible structures of the activation products using the model compounds of the precursors [17]. These activation products or compounds corresponding to the activation products in the P. stipticus fruiting bodies have not yet been found. Subsequently, Shimomura analyzed the interrelationships between the bioluminescence and the superoxide dismutase activities using six species of fungi, including $P$. stipticus (fruiting body and mycelium), and demonstrated that superoxide dismutase regulated the bioluminescence activity [18]. On the basis of these findings, Shimomura suggested that, unlike Airth's luciferin-luciferase reaction, $P$. stipticus bioluminescence was not a luciferin-luciferase type. Shimomura also suggested that superoxide anion triggered the bioluminescence reaction in the presence of molecular oxygen for generating light.

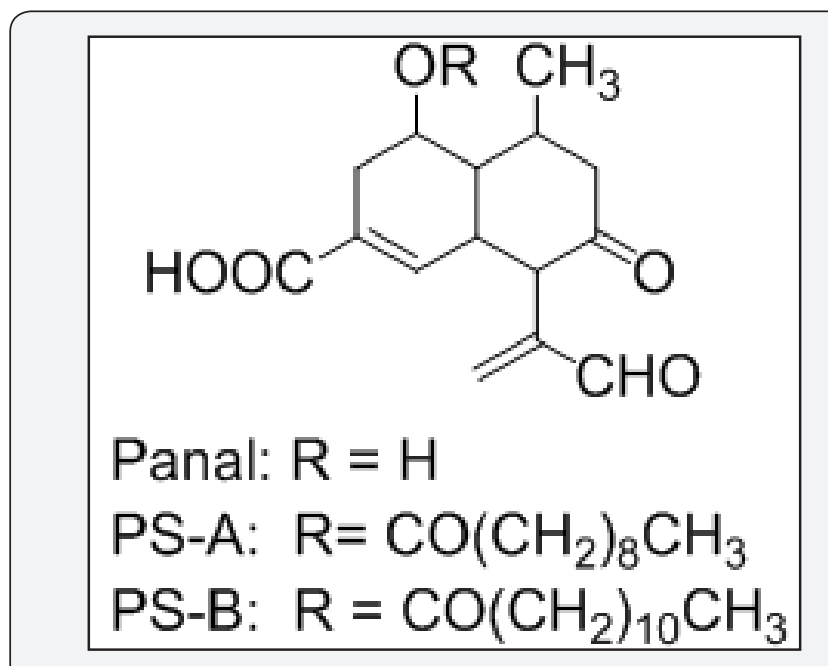

Figure 2 : Chemical structures of panal, PS-A, and PS-B isolated from $P$. stipticus by Shimomura.

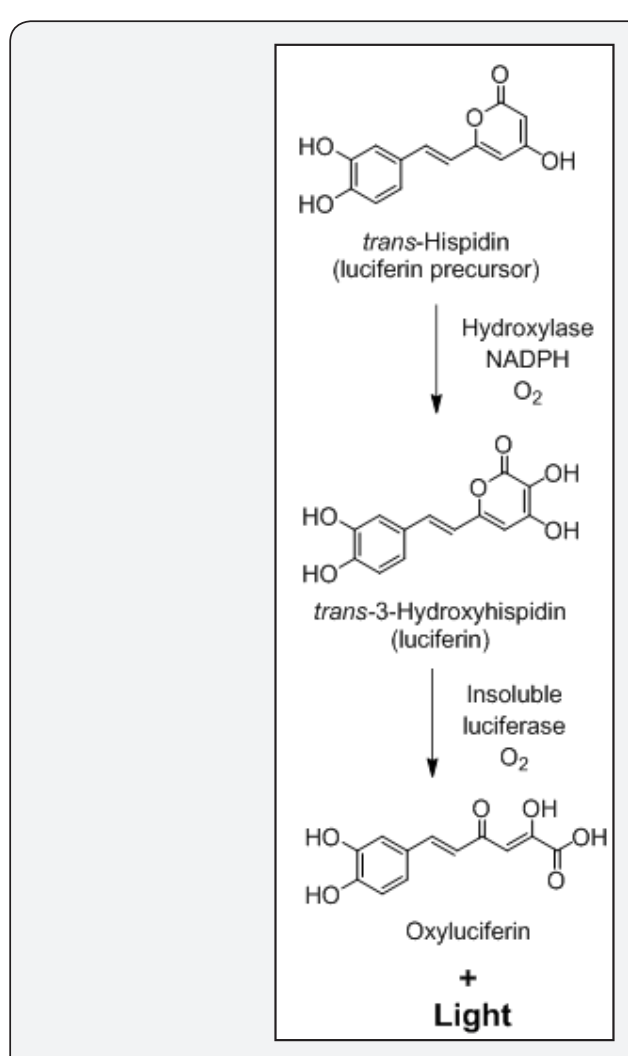

Figure 3 : Chemical mechanism underlying $N$. gardneri and bioluminescence proposed by Yampolsky's group [20,21].

In 2012, Oliveira et al. reported the hypothesis that 
all known bioluminescent fungal lineages share luciferinluciferase reaction shown by Airth's group [19]. In 2015, Yampolsky's group reported that and trans-3-hydroxyhispidin, which played as luciferin with water-insoluble luciferase, was produced from trans-hispidin with molecular oxygen and NADPH in the presence of water-soluble hydroxylase for mycelia bioluminescence of Neonothopanus nambi (Figure 3) [20]. Then, Yampolsky's group reported that and trans-3-hydroxyhispidin was oxidized to $(2 Z, 5 E)-6$-(3,4-dihydroxyphenyl)-2-hydroxy4-oxohexa-2,5-dienoic acid(oxyluciferin) as a light emitter in the presence of a luciferase-enriched protein fraction prepared from N. gardneri and N.nambi mycelium (Figure 3) [21]. Trans3-hydroxyhispidin is a strong "candidate" of luciferin in $N$. gardneri and N.nambi mycelia at the present time. Luciferase has been successfully extracted from N. gardneri and N.nambi mycelia and partially purified [21]. Therefore, it is distinct that these mycelia possess luminescence systems involving trans-3-hydroxyhispidin. In the future it is necessary to elucidate whether these luminescence systems generate true bioluminescence in the living N. gardneri and N.nambi mycelia or not, and whether similar bioluminescence systems involving trans-3-hydroxyhispidin exist in other bioluminescent fungi or not.

Although various fungal species have been used for chemical research of fungal bioluminescence over the years, mycelia were subject for the research, except for Shimomura's experiments above mentioned. Some researchers believe that bioluminescence mechanism for all bioluminescent fungi should be the same, and that chemical mechanism behind bioluminescence in mycelia and fruiting bodies is the same. Because bioluminescent fungus Mycena chlorophos (Figure 1), which was found in Southeast Asia [22] and can be cultivated in laboratory $[23,24]$, produces continuously bright light in the mycelium and pileus gills, in which light is produced in cell membrane [25], this species is a suitable for evaluating those hypotheses. Teranishi has been chemically investigating bioluminescence mechanism of this fungus using living tissue. Generally when fungal tissue is crushed, bioluminescence is immediately stopped. Because the crushed tissue losses the bioluminescence ability, the crushed tissue and extracts from the tissue cannot be used for luminescence assays. Recently Teranishi demonstrated that trans-4-hydroxycinnamic acid and trans-3,4-dihydroxycinnnamic acid in gills increased the bioluminescence intensity in living immature gills of M. chlorophos, which emitted weak light [26,27], and that other cinnamic acids shown in Figure 4 did not influence the original light emission. Moreover, the light emission before and after addition of trans-4-hydroxycinnamic acid ortrans-3,4-dihydroxycinnnamic acid to gills was inhibited by addition of trans-4-aminocinnnamic acid, whereas trans-2and trans-3-aminocinnnamic acids did not inhibit the light emission [28].These results indicate that trans-4- and/or 3,4-di-hydroxycinnamic acids contribute to bioluminescence in $M$. Chlorophos living gills, and that the hydroxy group at position C-4 intrans-cinnamic acid plays an important role for producing the energy for light emission. Although Teranishi reported that the combination of NADPH and hispid in was not essential for the production of bright light in the living gills [29], bioluminescence ability of trans-3-hydroxyhispidin yet remains to be elucidated. Investigation for understanding the chemical mechanism underlying bioluminescence observed in $M$. chlorophos gills and mycelia is in progress.

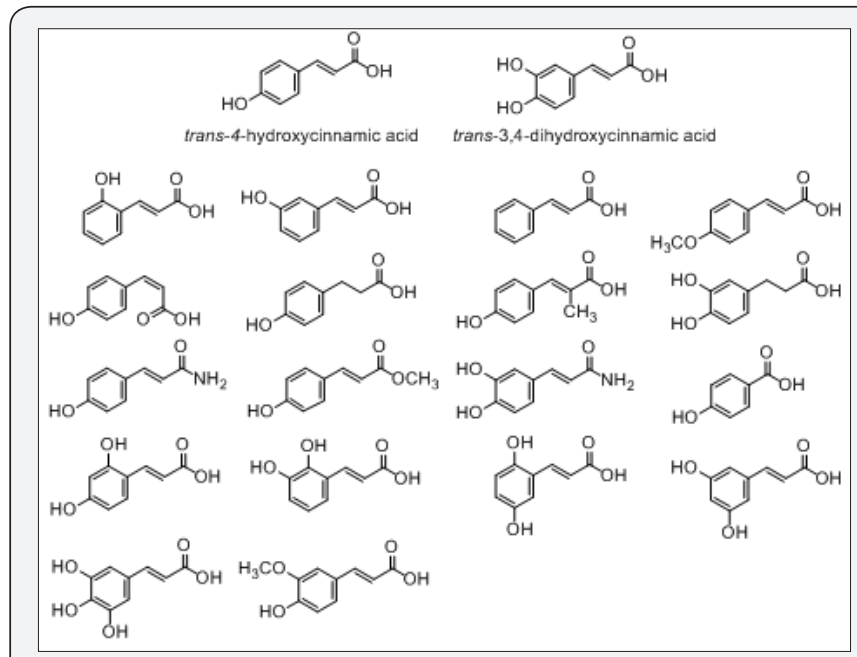

Figure 4 : Compounds used in the assay for gill bioluminescence of $M$. chlorophos $[26,27]$.

\section{References}

1. Shimomura O (2012) Bioluminescence: chemical principles and methods. World Scientific Publishing Co. Pte. Ltd., Singapore.

2. Oba Y, Schultz DT (2014) Bioluminescence: fundamentals and applications in biotechnology. 1. Springer-Verlag Berlin Heidelberg, Germany p. 4-14.

3. Esimbekova E (2014) Bioluminescence: fundamentals and applications in biotechnology. 1. Springer-Verlag Berlin Heidelberg, Germany pp. 67-197.

4. Desjardin DE, Perry BA, Stevani CV (2016) New luminescent mycenoid fungi (Basidiomycota, Agaricales) from São Paulo State, Brazil. Mycologia 108: 1165-1174.

5. O'Kane DJ, Lingle WL, Porter D, Wampler JE (1990) Localization of bioluminescent tissues during basidiocarp development in Panellus stypticus. Mycologia 82(5): 595-606.

6. Desjardin DE, Capelari M, Stevani C (2007) Bioluminescent Mycena species from São Paulo, Brazil. Mycologia 99(2): 317-331.

7. Desjardin DE, Oliveira AG, Stevani CV (2008) Fungi bioluminescence revisited. Photochem Photobiol Sci 7(2): 170-182.

8. Oliveira AG, Stevani CV, Waldenmaier HE, Viviani V, Emerson JM, et al. (2015) Circadian control sheds light on fungal bioluminescence. Current Biology 25(7): 964-968.

9. Weinstein P, Delean S, Wood T, Austin AD (2016) Bioluminescence in the ghost fungus Omphalotus nidiformis does not attract potential spore dispersing insects. IMA fungus 7(2): 229-234.

10. Boyle R (1668) Experiments concerning the relation between light and air in shining wood and fish. Philos Trans R Soc London 2: 581-600.

11. Airth RL, McElroy WD (1959) Light emission from extracts of luminous fungi. J Bacteriol 77(2): 249-250. 
12. Airth RL, Foerster GE (1960) Some aspects of fungal bioluminescence. J Cell Comp Physiol 56: 173-182.

13. Airth RL, Foerster GE (1962) The isolation of catalytic components required for cell-free fungal bioluminescence. Arch Biochem Biophys 97: 567-573.

14. Kuwabara S, Wassink EC (1966) Purification and properties of the active substance of fungal luminescence. In Bioluminescence in Progress. (Edited by Johnson FH and Haneda Y), Princeton University Press, Princeton, NJ. pp. 233-245.

15. Shimomura O (1989) Chemiluminescence of panal (a sesquiterpene) isolated from the luminous fungus Panellus stipticus. Photochem Photobiol 49: 355-360.

16. Shimomura O (1991) Superoxide-triggered chemiluminescence of the extract of luminous mushroom Panellus stipticus after treatment with methylamine. J Exp Botany 42(237): 555-560.

17. Shimomura $O$ (2012) Luminous fungi. In Bioluminescence: chemical principles and methods. World Scientific Publishing Co. Pte. Ltd., Singapore. pp. 275-308.

18. Shimomura 0 (1992) The role of superoxide dismutase in regulating the light emission of luminescent fungi. J Exp Botany 43(256): 15191525 .

19. Oliveira AG, Desjardin DE, Perry BA, Stevani CV (2012) Evidence that a single bioluminescent system is shared by all known bioluminescent fungal lineages. Photochem Photobiol Sci 11(5): 848-852.

20. Purtov KV, Petushkov VN, Baranov MS, Mineev KS, Rodionova NS, et al. (2015) The chemical basis of fungal bioluminescence. Angew Chem Int Ed 54(28): 8124-8128.

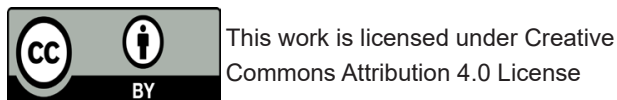

21. Kaskova ZM, Dörr FA, Petushkov VN, Purtov KV,Tsarkova AS, et al. (2017) Mechanism and color modulation of fungal bioluminescence. Sci Adv 3(4): e1602847.

22. Kobayashi Y (1937) Several luminous my comycetes from bonin islands. Bull Biogeogr Soc Japan 7: 1-7.

23. Niitsu H, Hanyuda N, Sugiyama Y (2000) Cultural properties of a luminous mushroom Mycena chlorophos. Mycoscience 41(6): 551-558.

24. Niitsu H, Hanyuda N (2000) Fruit-body production of a luminous mushroom, Mycena chlorophos. Mycoscience 41(6): 559-564.

25. Teranishi K (2016) Localization of the bioluminescence system in the pileus of Mycena chlorophos. Luminescence 31(2): 594-599.

26. Teranishi K (2016) Trans-p-hydroxycinnamic acid as a bioluminescence-activating component in the pileus of the luminous fungus Mycena chlorophos. Tetrahedron 72: 726-733.

27. Teranishi K (2017) Second bioluminescence-activating component in the luminous fungus Mycena chlorophos. Luminescence 32(2): 182189.

28. Teranishi K (2017) Inhibition of bioluminescence in the living gills of the luminous fungus Mycena chlorophos by trans-4-aminocinnamic acid. Biochem Biophys Res Commun 488(2): 335-339.

29. Teranishi K (2017) A combination of NADHP and hispidin is not essential for bioluminescence in luminous fungal living gills of Mycena chlorophos. Luminescence 32(5): 866-872.

\section{Your next submission with Juniper Publishers will reach you the below assets}

- Quality Editorial service

- Swift Peer Review

- Reprints availability

- E-prints Service

- Manuscript Podcast for convenient understanding

- Global attainment for your research

- Manuscript accessibility in different formats

( Pdf, E-pub, Full Text, Audio)

- Unceasing customer service

Track the below URL for one-step submission https://juniperpublishers.com/online-submission.php 